\title{
Morphine spinals: ICU or ward?
}

\author{
Quan C \\ Department of Anaesthesia, Chris Hani Baragwanath Academic Hospital,University of the Witwatersrand, South Africa \\ Corresponding author, email:barrowceleste@gmail.com
}

\section{History}

Josef Wang first described the use of intrathecal morphine in 1977. ${ }^{1}$ He injected 8 rats (weighing between 400-500 g) with $25 \mu \mathrm{g}$ intrathecal morphine. The tail-flick response was monitored. He concluded that intrathecal morphine might become a predictable modality of pain relief.

Yaksh followed 2 years later with a study looking at the use of intrathecal morphine in parturition in rats and rabbits. ${ }^{2}$ Gravid rats and rabbits had intrathecal catheters inserted. After the initiation of nest building, the rats were injected with 15, 45 and $100 \mu \mathrm{g}$ of intrathecal morphine and the rabbits with $80 \mu \mathrm{g}$. Analgesia was tested in the rats with a hot plate as well as a tail flick test. In rabbits it was tested with a hot probe. The animals were well analgesed and there was no difference compared to controls with the onset of delivery as well as the percentage of pups alive after 150 minutes. Alper in the editorial of the same journal in which Yaksh's article was published, described intrathecal morphine as "potentially revolutionary".2 Intrathecal morphine provided analgesia in gravid rats and rabbits, had no effect on parturition and seemed safe for both the mother and the child.

Josef Wang was at this stage, also exploring the use of intrathecal morphine for the treatment of intractable pain of inoperable cancer. $^{3}$

\section{Pharmacology}

Opioid receptors are found in the brain (periaqueductal grey matter, rostral ventral medulla and medial thalamic limbic system). In addition, opioid receptors are also found in the spinal cord (Rexed laminae II and V and substantia gelatinosa). Of the 4 opioid receptors, 3 ( $\mu, \mathrm{K}$ and $\delta$ ) are found in the spinal cord. ${ }^{4}$ Theoretically, because intrathecal morphine acts at specific opioid receptors it provides analgesia without actions on other sensory or motor functions. ${ }^{5}$

Opioids can be classified according to their solubility. Morphine is a hydrophilic opioid and remains in the cerebrospinal fluid for longer than the lipophilic opioids (fentanyl and sufentanil). This is advantageous as this allows the drug to remain in the intrathecal space as it crosses the dura poorly. This results in a prolonged duration of action and little drug should be found in maternal and foetal blood. ${ }^{5}$

\section{Effectiveness}

It has been shown that for postoperative pain relief and time to first analgesic request following spinal anaesthesia, the duration of intrathecal bupivacaine ranges from 90-190 minutes and is up to $184(+/-20)$ minutes for intrathecal fentanyl and bupivacaine. ${ }^{6}$ Intrathecal morphine (in a dose of $0.1 \mathrm{mg}$ ) results in an least 11 hours of effective analgesia and a significant reduction in postoperative analgesic requirements. ${ }^{7}$

\section{Adverse effects}

Adverse effects include pruritus, nausea and vomiting and respiratory depression. ${ }^{7}$ Of these, respiratory depression is the most feared. The difficulty in reviewing the literature is that the term "respiratory depression" has no clear definition. ${ }^{8}$ Consequently determining the exact incidence is not a perfect science.

The reported incidence of respiratory depression ranges from $0-0.9 \% .^{9}$

The incidence of respiratory depression correlates with the dose of intrathecal morphine. Meta-analysis indicates a reduced frequency of hypoxaemia when lower doses (vs. higher doses) of single-shot intrathecal opioids are used. ${ }^{10}$ The optimal dose (balancing effective analgesia and a low incidence of adverse effects) most probably is between $75-150 \mu \mathrm{g} .{ }^{11}$

In one study, neither $100 \mu \mathrm{g}$ nor $250 \mu \mathrm{g}$ intrathecal morphine affected minute ventilation or the ventilator responses to $\mathrm{CO}_{2^{\prime}}$ whereas both measurements were depressed for 3 hours after 8 mg subcutaneous morphine. ${ }^{12}$

Bailey et al. explored the effects of $300 \mu \mathrm{g}$ of intrathecal morphine versus $0.14 \mathrm{mg}$ per kilogram of body weight on ventilator drive. ${ }^{13} \mathrm{He}$ found that the depression of the ventilator response to hypoxia after the administration of intrathecal morphine is similar in magnitude to, but longer lasting than, that after an equianalgesic dose of intravenous morphine.

The mechanisms of respiratory depression include9:

- Vascular uptake by the epidural or subarachnoid venous plexuses and circulation to brainstem respiratory center.

- Arachnoid penetration and movement into the spinal cord. 
- Rostral spread via the aqueous cerebrospinal fluid to the brainstem.

- Rostral spread via direct perimedullary vascular channels.

\section{Patients at risk ${ }^{9}$}

- Obstructive sleep apnoea

- Morbid obesity

- Elderly

- Cardiopulmonary disease

- Preoperative opioid tolerance

- Hypermagnesemia (in obstetric patients)

\section{Guidelines}

The American Society of Anesthesiologists has published guidelines for the post operative monitoring of patients who have had intrathecal monitoring. ${ }^{10}$ This should be done hourly for the first 12 hours and then 2 hourly for the next 12 hours. Monitoring should include respiratory rate as well as level of consciousness. Pulse oximetry is not more sensitive than clinical monitory. Capnography is sensitive but has severe practical limitations.

\section{Conclusions}

Intrathecal morphine has the advantages of:

\section{Simplicity}

2. Reliability

3. Prolonged duration without a catheter in situ

With all interventions in anaesthesia, one must weigh up the benefits versus the risk. The most dreaded risk of intrathecal morphine is that of respiratory depression. This risk is dosedependent. Doses of $100 \mu \mathrm{g}$ are effective for analgesia with very little risk of respiratory depression. However, respiratory depression is potentially fatal. Therefore the patient at risk for respiratory depression must be recognized and adequate monitoring put in place. Over-sedation and respiratory depression should be treated proactively.

If a patient does not have any riskfactors for respiratory depression and receives low dose intrathecal morphine $(<300 \mu \mathrm{g})$, it would seem that the risk for respiratory depression is the same or even less than the same patient receiving parenteral opioids. There are no data to support the use for extended monitoring of patients receiving low dose intrathecal morphine. ${ }^{14}$

In conclusion, if the patient has no risk factors for respiratory depression and you are happy to prescribe parenteral opioids in the ward, you should be happy to send the patient to the ward post low dose $(<300 \mu \mathrm{g})$ morphine spinal. Sound evidencebased medicine must be followed as opposed to dogma.

\section{References}

1. Wang J. Analgesic effect of Intrathecally Administered Morphine. Reg Anesth Pain Med. 1977;2(3):3, 8.

2. Yaksh $T$, Wilson $P$, Kaiko $R$, Inturris iC. Analgesia produced by a Spinal Action of Morphine and Effects upon Partutition in the Rat. Anesthesiology. 1979;51(5):386-92.

3. Wang J, Nauss L, Thomas JE. Pain relief by intrathecally applied morphine in man. Anesthesiology. 1979;50:149-51.

4. Milner A, Welch E. Applied Pharmacology in Anaesthesiology and Critical Care Centurion: Medpharm Publications; 2012.

5. Alper M. Intrathecal Morphine: A New Method of Obstetric Analgesia. Anesthesiology. 1979;51(5):378-9.

6. Wagner J. Time until first analgesic requirement, post caesarean section under spinal anaesthesia in HIV-positive patients at Chris Hani Baragwanath Hospital: University of the Witwatersrand; 2010.

7. Dahl J, Jeppesen I, Jorgensen H, Wetterslev J, Moiniche S. Intraoperative and postoperative analgesic efficacy and adverse effects of Intrathecal opioids in patients undergoing cesarian section with spinal morphine. Anesthesiology. 1999;91(5):1919-27.

8. Ko S, Goldstein D, VanDenKerkhof E. Definitions of "respiratory depression" with intrathecal morphine postoperative analgesia: a review of the literature. Can J Anaesth. 2003;50(7):679-88.

9. Carvalho B. Respiratory Depression After Neuraxial Opioids in the Obstetric Setting. Anesth Analg. 2008;107(3):956-61.

10. American Society of Anesthesiologists Task Force on Neuraxial Opioids. Practice Guidelines for the Prevention, Detection, and Management of Respiratory Depression Associated with Neuraxial Opioid Administration. Anesthesiology. 2009;110(2):1-13.

11. Ozbek H, Deniz M, Erakgun A, Erhan E. Comparison of 75 and $150 \mu \mathrm{g}$ doses of intrathecal morphine for postoperative analgesia after transurethral resection of the prostate under spinal anesthesia. J Opioid Manag. 2013;9(6):415-20.

12. Abboud T, Dror A, Mosaad $P$, et al. Mini-Dose Intrathecal Morphine for the Relief of Post-Cesarean Section Pain. Anesth Analg. 1988;67:137-43.

13. Bailey P, Lu J, Pace N, et al. Effects of intrathecal morphine on the ventilatory response to hypoxia. N Engl J Med. 2000;343(17):1228-34.

14. Gehling M, Tryba M. Risks and side-effects of intrathecal morphine combined with spinal anaesthesia: a meta-analysis. Anaesthesia. 2009;64:643-51. 